\title{
An Orbital-free Molecular Dynamics Study of Static and Dynamic Properties of Liquid Sn
}

\author{
G. M. Bhuiyan ${ }^{1}$, L.E. González ${ }^{2}$ and D.J. González ${ }^{2}$ \\ 1 Department of Theoretical Physics, University of Dhaka, Dhaka-1000, Bangladesh, \\ 2 Departamento de Fisica Teórica, Facultad de Ciencias, Universidad de Valladolid, Valladolid, SPAIN
}

\begin{abstract}
The static and dynamic properties of liquid Sn at two different thermodynamic states have been studied from the Orbital Free Ab-Initio Molecular Dynamics (OFAIMD) simulation method. This method is based on the density functional theory (DFT) of Hohenberg and Kohn. The exchange and correlation interaction is described here with the local density approximation (LDA). We, in this study, have developed a pseudopotential model to describe the external non-Coulombic interaction acting on an electron; this is an essential ingredient of the theory. We have studied several static and dynamic properties of the system. The calculated results are found to be good in agreement with the available experimental data. They are also found to be of similar accuracy as those of other ab-initio studies.
\end{abstract}

Keyworkds: Static and dynamic properties, liquid metals, OFAIMD, DFT, LDA, Pseudopotential.

\section{Introduction}

The first principles calculations along with Molecular Dynamics (MD) simulation techniques have become an essential way to study different properties of condensed matters [1-4]. Most of the first principles methods are based on the Density Functional Theory [5-6]. Since the microscopic details of the orbital are involved in the Kohn-Sham ab-initio molecular dynamics (KS-AIMD), calculations are computationally very expensive, and this limits the size of the systems (one or two hundred particles) under study. However, some of these constraints may be greatly reduced by the so called orbital free ab initio MD (OF-AIMD) simulation method [3,7]. In the OF-AIMD method the electronic orbitals of KS formulation are disposed of by using only the valence electron density, expanded in a plane wave basis set, which greatly reduces the number of variables describing the electronic state, and thus enables to study larger sample and for longer simulation time (tens of picoseconds). We report, in the present work, OF-AIMD simulation results for the static and dynamic properties of liquid Sn at two different thermodynamic states characterized by temperatures of $\mathrm{T}=573 \mathrm{~K}$ and $1275 \mathrm{~K}$.

$\mathrm{Sn}$ in the liquid phase displays some characteristics features in their structural properties. For example, the static structure factor $S(q)$ displays a shoulder, although small, at high q-side of the main peak, similar, although less marked, to liquid $\mathrm{Si}$ and liquid Ge, and a coordination number $\left(\mathrm{C}_{N}\right)$ around 9-10 unlike $\mathrm{Si}$ and $\mathrm{Ge}$ that have $\mathrm{C}_{N}$ around 6. Liquid $\mathrm{Sn}$ for its characteristic features attracted attention of theoreticians $[8,12-16]$ and experimentalists [8-11]. But to the best of our knowledge, this is the first application of the OF-AIMD method with a relatively large sample.

Within the Hohenberg and Kohn theory of DFT [5] the external potential is a local function generated by the positive ions and, it is normally treated by a pseudopotential. This local pseudopotential plays an essentially important role in determining the energy as well as the forces acting on ions. In the present study we initially tried with different model pseudopotentials for Sn available in the literature [12-14], but none of them could produce static and dynamic properties together as experiments suggest. Finally, we have developed a simple model (see below) to describe the electron-ion interaction and found that it works sufficiently well in predicting different physical properties at several thermodynamic states.

\section{Theories}

Different theories relevant to the OF-AIMD simulation method, namely, the density functional theory with an approximated kinetic energy functional, and pseudopotential are described in this section.

\subsection{Density Functional Theory}

Within the density functional theory (DFT) the total energy functional may be written as,

$$
E[n]=T_{S}[n]+E_{H}[n]+E_{X C}[n]+E_{\text {ext }}[n]
$$


where, $T_{S}[\mathrm{n}]$ is the kinetic energy, $E_{H}[\mathrm{n}]$ the Hartree electrostatic energy, $E_{X C}[\mathrm{n}]$ exchange and correlation energy and $E_{\text {ext }}[\mathrm{n}]$ the energy due to the external potential. Within an average density model, the kinetic energy functional for non-interacting electrons may be written as [3]

$$
T_{S}=T_{W}+T_{\beta}
$$

with

$$
T_{W}=\frac{1}{8} \int d \bar{r} \frac{|\nabla n(r)|^{2}}{n(r)}
$$

and

$$
\begin{gathered}
T_{\beta}=\frac{3}{10} \int d^{3} r n(\bar{r})^{\left(\frac{5}{3}-2 \beta\right)} \tilde{k}(\bar{r})^{2} \\
\tilde{k}(\bar{r})=\left(2 k_{F}^{(0)}\right)^{3} \int d \bar{r}^{\prime} k\left(\bar{r}^{\prime}\right) w_{\beta}\left(2 k_{F}^{(0)}\left|\bar{r}-\overline{r^{\prime}}\right|\right)
\end{gathered}
$$

where

$$
k\left(\overline{r^{\prime}}\right)=\left(3 \pi^{2}\right)^{\frac{1}{3}} n\left(\overline{r^{\prime}}\right)^{\beta},
$$

and $k_{F}^{(0)}$ is the Fermi wave vector corresponding to a mean electron density $n_{e}=\frac{N_{e}}{V}$, and $w_{\beta}$ is a weight function chosen so that both the linear response theory and the Thomas-Fermi limits are correctly recovered.

\subsection{Pseudopotential}

In the orbital free MD approach the electron density is a variable, so non-local pseudopotentials cannot be used. A local pseudopotential must therefore be developed that includes an accurate description of the electronic structure in the physical circumstance of interest. In this situation we have proposed a new local pseudopotential of the following form,

$$
V_{p s}(r)= \begin{cases}A+B e^{-\frac{r}{a}} & \text { for } r<R_{c} \\ -\frac{Z e^{2}}{r} & \text { for } r>R_{c}\end{cases}
$$

where $A$ and $B$ are constants, $R_{c}$ is the core radius and $a$ is the softness parameter. Following the work of Abarenkov and Heine [15] we set a condition that the logarithmic derivative of the potential inside and outside the core exactly matches at the core radius. This permits the elimination of $B$ as a parameter. The values of the constants for liquid Sn are specified in table 1 .

Table 1. Values for the pseudopotential parameters. The same values are used for the two temperatures considered.

\begin{tabular}{|c|c|c|}
\hline$A$ (a.u.) & $a$ (a.u.) & $R_{c}$ (a.u.) \\
\hline-0.08 & 0.80 & 1.55 \\
\hline
\end{tabular}

\section{Results and Discussions}

The results of calculations for static and dynamic properties of liquid Sn from the OF-AIMD simulation are presented in this section. In the simulation we have used 500 atoms in a cubic cell with periodic boundary conditions. The plane wave expansion is truncated at a cut off energy $E_{c u t}=20$ Ryd. The energy minimization with respect to the Fourier coefficients of the expansion is performed every time step leading to the ground state electronic density and energy.

Figure 1(a) shows the non-Coulombic electron-ion interaction in $q$ space. It is seen that the magnitude of the interaction is maximum in the long wavelength limit. The magnitude decreases with increasing $q$ and becomes slightly oscillatory after $q>4 \AA^{-1}$ which is also observed with other model potentials [12-14]. The value of the present pseudopotential at $q=0$ is clearly smaller than that of Fiolhais pseudopotential, but comparable to Ashcroft and Bretonnet-Silbert (BS) ones. On the other hand, the $q$-range where the pseudopotential decays is much greater for the BS pseudopotential, the other three being comparable. Our model shows in fact the shortest range. We believe that these features made the present model suitable for its application to liquid Sn.

\subsection{Static properties}

The simulation allows to have a direct evaluation of static structure factor $S(q)$ and its counterpart in real space, the pair correlation function $g(r)$ at two different thermodynamic states. Figure 1(b) shows the pair correlation function $g(r)$. A comparison with the available x-ray diffraction data [10] shows a good agreement both in phase of oscillations and magnitude except at the level of the principal peak, where simulation values are slightly larger than the corresponding experimental ones, in particular, at $1273 \mathrm{~K} . g(r)$ provides information about the short range order in the liquid. For $573 \mathrm{~K}$ the position of the principal peak of $g(r)$ is at $3.107 \AA$ and the ratio of the positions of the first and second peaks is 2.02 whereas for simple liquid metals the ratio lies in the range 1.84-1.93 [10]. In order to evaluate the coordination number, $\mathrm{C}_{N}$, we integrated the radial distribution function $4 \pi \rho r^{2} g(r)$ up to the position of the first minimum which is $3.93 \AA$ for both temperatures. The results are given in Table 2 and are very close to other ab initio results [8].

Figure 1(c) shows $S(q)$ as a function of $q$ at $\mathrm{T}=573$ $\mathrm{K}$ and $\mathrm{T}=1273 \mathrm{~K}$. The figure shows the main characteristics of $S(q)$ for liquid $\mathrm{Sn}$ : an asymmetric main peak located at $q_{p}=2.22 \AA^{-1}$ and a hump at the right of $q_{p}$, located at $q_{h}=3.093 \AA^{-1}$. Comparison with the x-ray diffraction data [10] at $573 \mathrm{~K}$ shows an excellent agreement for the whole $q$ range except at the level of principal peak where the peak value is slightly overestimated. Similar agreement is also found at a higher temperature $\mathrm{T}=1273 \mathrm{~K}[30]$. We note here that the 
level of accuracy of the OF-AIMD results is similar to that of other AIMD simulation study [8].

(a)

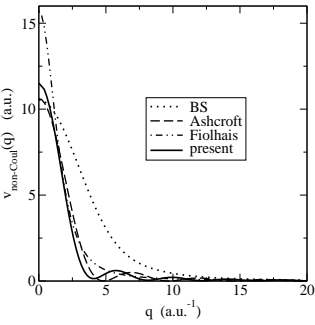

(b)
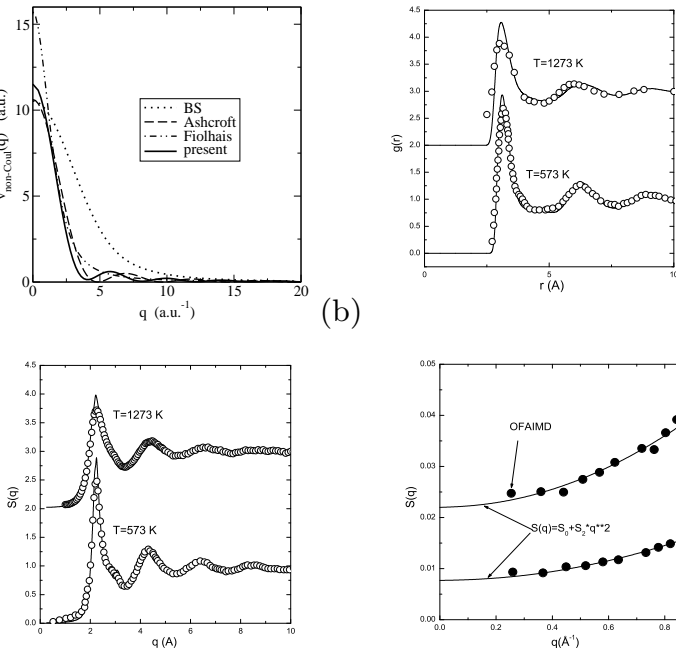

(c)

(d)

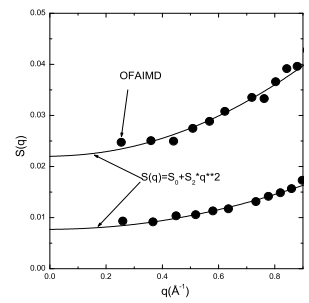

Fig. 1. (a) Bare non-Coulombic electron-ion interaction in momentum space for different pseudopotential models. (b) Pair correlation functions at $573 \mathrm{~K}$ and $1273 \mathrm{~K}$. Line: simulation data, circles: experimental values. (c) Static structure factor S(q), same meaning of symbols. (d) Least square fit to the $\mathrm{S}(\mathrm{q})$ at low $\mathrm{q}$.

It is worth noting that the small hump in $S(q)$ is microscopically connected to the existence of bonds corresponding to pairs of atoms closer than the average neighbour distance in the liquid [8]. A closer look at $S(q)$ at a higher temperature $\mathrm{T}=1273 \mathrm{~K}$ reveals that the hump disappears at high temperature. This is due to the thermal broadening of the main peak, that leads to covering small features like the hump.

The static structure factor $S(q)$ connects to thermodynamics in the long wavelength limit through the isothermal compressibility $\chi_{T}: S(0)=n k_{B} T \chi_{T}$. In order to determine $S(0)$ we fit a parabolic expression for structure factor $S(q)=S_{0}+S_{2} q^{2}$ to the OF-AIMD data at low $q$ (see figure $1(\mathrm{~d})$ ). Values of $\chi_{T}$ calculated are listed in Table 2 and are good in agreement with experimental data.

\subsection{Dynamic properties}

Dynamic properties of liquid Sn at two different thermodynamic states characterized by $\mathrm{T}=573 \mathrm{~K}$ and $1273 \mathrm{~K}$ are presented in this section.

\subsubsection{Single particle dynamics}

Information about different atomic transport properties of liquid systems can be extracted from the
Table 2. Results obtained for the coordination number $C_{N}$ and isothermal compressibility $\chi_{T}$ at the two thermodynamic states considered. Values in the parenthesis are experimental data [16], and in the curly bracket ab-initio results obtained with 205 particles [8].

\begin{tabular}{|c|c|c|c|}
\hline $\begin{array}{c}\mathrm{T} \\
(\mathrm{K})\end{array}$ & $\begin{array}{c}n \\
\left(\AA^{-3}\right)\end{array}$ & $C_{N}$ & $\begin{array}{c}\chi_{T} \times 10^{11} \\
\left(\mathrm{~N}^{-1} \mathrm{~m}^{2}\right)\end{array}$ \\
\hline 573 & 0.035 & $9.2\{9.4\}$ & $2.78(2.71)$ \\
1273 & 0.032 & $8.2\{8.5\}$ & 3.80 \\
\hline
\end{tabular}

simulations. The normalized velocity autocorrelation function (VACF) of a tagged ion in the fluid, $Z(t)$ is defined as $Z(t)=\langle\bar{v}(0) \cdot \bar{v}(t)\rangle /\left\langle v^{2}\right\rangle$ where $v(0)$ and $v(t)$ are the velocities at reference and after an interval $t$ respectively. $\langle\ldots\rangle$ denote the ensemble average. Figure 2(a) shows $Z(t)$ as a function of $t$ for $\mathrm{T}=$ $573 \mathrm{~K}$ and $1273 \mathrm{~K}$. At $573 \mathrm{~K}, Z(t)$ exhibits the typical backscattering behaviour with a first minimum at $t=0.189 \mathrm{ps}$. However, the back scattering is much less at higher temperature $\mathrm{T}=1273 \mathrm{~K}$. The self diffusion coefficient, $D$, of an ion can be found by integrat$\operatorname{ing} Z(t), D=\frac{k_{B} T}{M^{*}} \int_{0}^{\infty} Z(t) d t$ or from the slope of the mean square displacement $\delta R^{2}=<\left|R_{1}(t)-R_{1}(0)\right|^{2}>$ of a tagged ion in the fluid. From the first method we have found $D=0.20 \AA^{2} / \mathrm{ps}$ at $\mathrm{T}=573 \mathrm{~K}$. This value is fairly close to the microgravity data $D_{\operatorname{Exp}}=0.25$ $\AA^{2} /$ ps [17]. At the higher temperature $\mathrm{T}=1273 \mathrm{~K}$ we found $D=0.79 \AA^{2} /$ ps whereas the experimental data available is $D_{\operatorname{Exp}}=1.14 \AA^{2} / \mathrm{ps}$ [18]. We note here that one AIMD simulation gave $D=1.10[8]$ and another gave $D=0.78 \AA^{2} / \mathrm{ps}[19]$.

\subsubsection{Collective dynamics}

The collective dynamics in a liquid system are generally described through an intermediate scattering function which contains relevant information over both length and time scales. Its time Fourier Transform is the dynamic structure factor, $S(q, \omega)$, which has a direct connection to the inelastic neutron or x-ray scattering data. We find that $S(q, \omega)$ shows well defined side peaks, which bears indication of collective density excitations, up to $q=1.214 \AA^{-1}$. Figures 2 (b-c) show $S(q, \omega) / S(q)$ compared with experimental data $[20,21]$ when the experimental resolution function is ignored (dashed lines) or taken into account through convolution (solid line), the latter showing a better agreement with experiment. The adiabatic sound velocity obtained from the position of side peak is $c_{s}=2344$ $\mathrm{m} / \mathrm{s}$ at $\mathrm{T}=573 \mathrm{~K}$. At a higher temperature $\mathrm{T}=1273$ $\mathrm{K}$ we have also observed side peaks and, the velocity of sound obtained from them was found to be $c_{s}=2244 \mathrm{~m} / \mathrm{s}$. The corresponding experimental values are $c_{s}=2448 \mathrm{~m} / \mathrm{s}$ at $573 \mathrm{~K}$ and $2284 \mathrm{~m} / \mathrm{s}$ at $1273 \mathrm{~K}$ [20-21]. The agreement with the experimental data is favourably good. From the positions of the side 
(a)
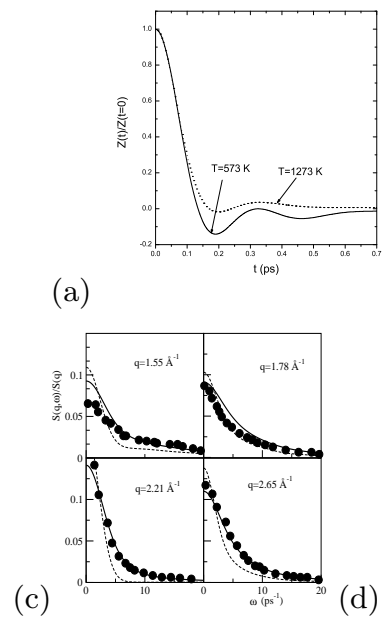

(b)
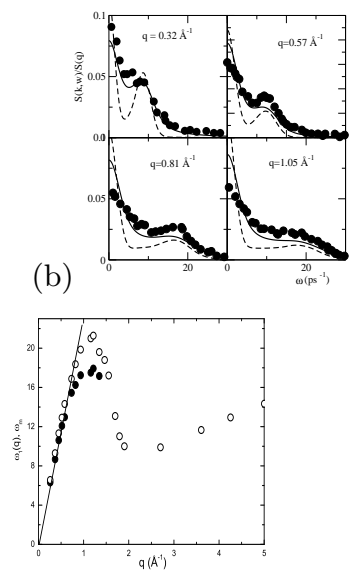

Fig. 2. (a) Velocity autocorrelation function. (b-c) Dynamic structure factors $S(q, \omega)$ compared with the experimental data at $\mathrm{T}=573 \mathrm{~K}$.Full circles for experimental data, dashed and solid line OFAIMD results before and after convolution, respectively, with experimental resolution function. (d) Dispersion relation, closed circles obtained from the side peak frequency and open circles from the peak positions of the longitudinal current correlation function.

peaks, $\omega_{m}$, the dispersion relation of the density fluctuations has been obtained. This dispersion relation is demonstrated in figure $2(\mathrm{~d})$ for $\mathrm{T}=573 \mathrm{~K}$. The longitudinal dispersion relation obtained from the maximum of the longitudinal current correlation function, $J_{l}(q, \omega)=\omega^{2} S(q, \omega)$ is also shown in the same figure. We note here that in the hydrodynamic limit, i.e at $q \rightarrow 0$ the slope of the dispersion relation curve gives the adiabatic sound velocity. The bulk velocity found in this way was $2328 \mathrm{~m} / \mathrm{s}$. This is very close to the experimental value [20] at $523 \mathrm{~K}$.

\section{Conclusions}

An orbital free ab-initio molecular dynamics simulation is performed for liquid Sn at two different thermodynamic states characterized by temperatures $\mathrm{T}=573$ $\mathrm{K}$ and $1273 \mathrm{~K}$. This OFAIMD method requires as an essential input a pseudopotential to describe the nonCoulombic electron ion interaction. Results obtained from the OFAIMD method along with our proposed pseudopotential agree very well with the available experimental data for both static and dynamic properties. In particular, the present approach can describe a fine structural feature like the hump in $S(q)$ successfully. The experimental dynamic structure factor is also well accounted for with the pseudopotential constructed in this work. Calculations of static and dynamic properties for liquid noble and transition metals, using this type of pseudopotential, are in progress and preliminary results are found to be very encouraging.

\section{Acknowledgments}

LEG and DJG acknowledge the support of MCINN and FEDER (grant FIS2008-02490/FIS) and JCyL (grants VA068A06 and GR120). GMB acknowledges the University of Valladolid, Spain, for offering him a fellowship without whose support this work would have been impossible. He is personally indebted to Professor D J Gonzalez and L E Gonzalez for their kind help and hospitality during his stay there.

\section{References}

[1] R. Car and M. Parrinello, Phys. Rev. Letts. 55, 2471 (1985).

[2] F. Shimojo, Y. Zempo, K. Hoshino, and M. Watabe, Phys. Rev. B 52, 9320 (1995).

[3] D. J. Gonzalez, L.E. Gonzalez, J. M. Lopez, and M. J. Stott, Phys. Rev. B 65, 184201 (2002).

[4] D. J. Gonzalez, L. E. Gonzalez, J. M. Lopez, and M. J. Stott, J. Chem. Phys. 115, 2373 (2001).

[5] P. Hohenberg W. Kohn, Phys. Rev. 136 B864 (1964). [6] W. Kohn and L. J. Sham, Phys. Rev. 140, A1133 (1965).

[7] D. J. Gonzalez, L. E. Gonzalez, and M. J. Stott, Phys. Rev. Lett. 94, 077801 (2005).

[8] L. Calderin, D. J. Gonzalez, L. E. Gonzalez and J. M. Lopez, J. Chem. Phys. 129, 194506 (2008).

[9] F. Zahid, G. M. Bhuiyan, S. Sultana, M. A. Khaleque, R. I. M.A. Rashid, and S. M. M. Rahman, Phys. Stat. Sol. (b) 215, 987 (1999).

[10] Y. Waseda, The Structure of Non-Crystalline Materials (McGraw-Hill, New York, 1980).

[11] S. Hosokawa, C. Pilgrim and F. Demmel, J. NonCryst. Solids 353,3122 (2007).

[12] N. W. Ashcroft, Phys. Lett. 23, 48 (1967).

[13] J. L. Bretonnet and M. Silbert, Phys. Chem. Liq. 24, 169 (1992).

[14] C. Fiolhais, J. P. Perdew, Q. Armster, J. M. MacLaren, and M. Brajczewska, Phys. Rev. B 51, 14001 (1995-II).

[15] I. V. Abarenkov and V. Heine, Phil. Mag. 12, 529 (1965).

[16] M. Shimoji, Liquid Metals, Academic press London, p.26 (1977).

[17] G. Frohberg, Material Sciences in Space (Springer, Berlin, 1986); G. Frohberg, K. H. Kraatz, and H. Weber, in proceedings of the 6th European Symposium on Material Sciences under microgravity conditions, Bordeaux (1986).

[18] A. Bruson and M. Gerl, Phys. Rev. B 21, 5447 (1980).

[19] T. Itami, S. Munejiri, T. Masaki, H. Aoki, Y. Ishii, T. Kamiyama, Y. Senda, F. Shimojo, and K. Hoshino, Phys. Rev. B 67, 064201 (2003).

[20] U. Balucani and M. Zoppi, Dynamics of the Liquid State (Clarendon, Oxford, 1994). 